\title{
Conformations of Dinucleoside Monophosphates in Relation to Duplex DNA Structures
}

\author{
SHASHIDHAR N. RAO ${ }^{*}$ and V. SASISEKHARAN ${ }^{\dagger}$ Molecular \\ Biophysics Unit, Indian Institute of Science, \\ Bangalore 560 012, India
}

\begin{abstract}
Mononucleotide conformations are important in understanding the structural aspects of nucleic acids and polynucleotides. In order to study the influence of stacking interactions between adjacent bases in a polynucleotide on the preferred conformations of mononucleotides, conformational energy calculations have been carried out on dinucleoside monophosphate fragments. Four base sequences- $d(A p T), d(T p A), d(C p G)$, and $\mathrm{d}(\mathrm{GpC})-$ have been analyzed in the framework of helical structures. Flexibility of the furanose ring has been incorporated in the investigations. Energetically favored conformers of the four compounds correspond to a variety of left- and right-handed uniform helical structures, similar to those of the commonly observed polymorphous forms. Implications of these investigations on the further understanding of double-helical polynucleotide conformations are briefly discussed.
\end{abstract}

\section{INTRODUCTION}

Conformational analysis of $3^{\prime}$ - and 5 '-mononucleotides constitute an important step in the structural elucidation of nucleic acids and polynucleotides. Both theoretical (conformational energy calculations) ${ }^{1-4}$ and experimental (nmr, ORD-CD, x-ray crystallography) ${ }^{5-8}$ methods have been employed in the analysis of these monomeric units. In our laboratory, as a part of the model-building studies on polynucleotides, conformational energy calculations (using classical potential functions) have been carried out on $3^{\prime}$ - and $5^{\prime}$-mononucleotides - $^{9,10}$ incorporating flexibility of the furanose ring. These calculations highlight the significance of sugar geometries, glycosidic torsion $(\chi)$, and $\mathrm{C4}^{\prime}-\mathrm{C5}^{\prime}$ torsion $(\gamma)$ in relation to energetically favorable conformations of nucleotides. Also, it has been indicated that of the conformational parameters defining a nucleotide repeat unit, the sugar pucker and the glycosidic orientation are the two most significant ones in determining the handedness of base-paired double-helical structures of polynucleotides. ${ }^{11}$

Conformational analysis of dinucleoside monophosphates (Fig. 1)

+ To whom correspondence should be addressed

* Present address: School of Pharmacy, Department of Pharmaceutical Chemistry, University of California, San Francisco, CA 94143. 


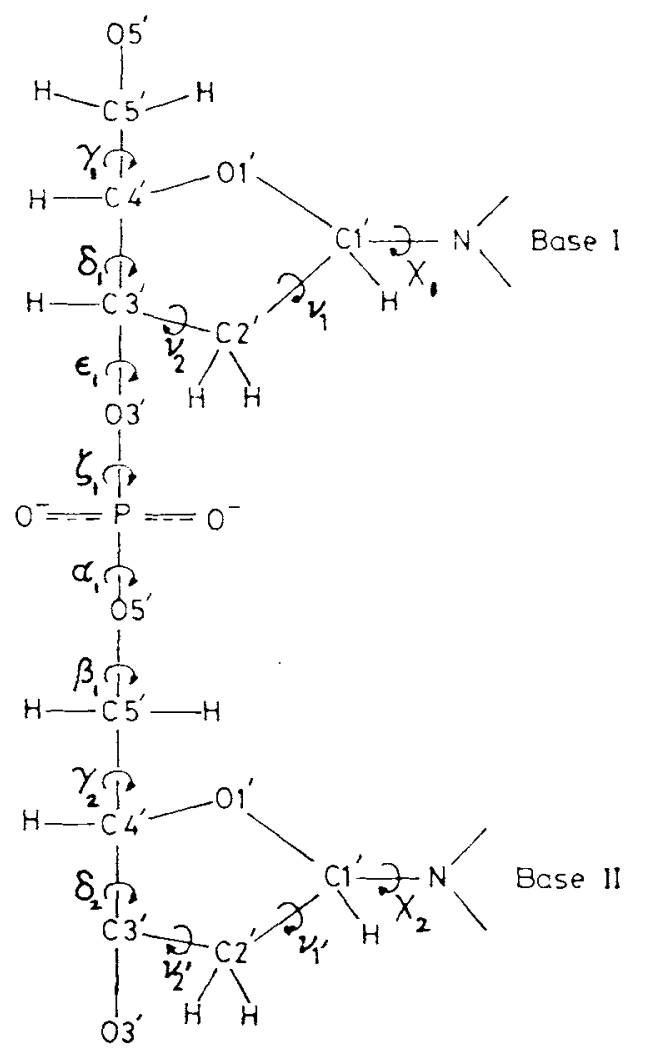

Fig. 1. The Dinucleoside monophosphate mioety used in the present study. The symbols used for the backbone, endocyclic, and glycosidic torsions are as recommended by the IUPAC-IUB JCBN (Ref. 40).

constitute the next important step in our understanding of polynucleotide structures. This moiety embodies base-base stacking interactions, in addition to the base-sugar, base-phosphate, and sugarphosphate interactions present in mononucleotides. Thus, the dinucleoside monophosphates have the essential conformational attributes of a polynucleotide chain. Therefore, it is of interest to determine how the energetically preferred conformations of the mononucleotides are affected by the presence of base--base stacking interactios. As a part of the larger program to analyze the energetic stabilities of regular helical polynucleotides with Watson-Crick base pairs and mononucleotide repeat units, the present investigations have been confined to the conformations of dinucleoside monophosphates meaningful from the viewpoint of helical structures. In particular, $(\mathrm{A}+\mathrm{T})$-and $(\mathrm{G}+\mathrm{C})$ containing sequences have been looked into with a view to examining their intrinsic stabilities in helical polynucleotides. It may, however, be noted that the calculations do not aim merely at obtaining all possible sets of energetically favorable conformations of dinucleoside 
monophosphates, but only those relevant to regular helical structures.

Since the present investigations have been confined to the framework of helical structures of polynucleotides only, the two glycosidic torsions and the sugar puckers have been kept the same. Thus, the conformations of the dinucleoside monophosphates are defined by $\chi$ and the backbone torsion angles $\alpha\left(\mathrm{P}^{-} \mathrm{O} 5^{\prime}\right), \beta\left(\mathrm{O}^{\prime}-\mathrm{C5}^{\prime}\right), \gamma\left(\mathrm{C}^{\prime}-\mathrm{C}^{\prime}\right), \epsilon\left(\mathrm{O}^{\prime}-\right.$ $\left.\mathrm{C}^{\prime}\right)$, and $\zeta\left(\mathrm{O3}^{\prime}-\mathrm{P}\right)$ and the sugar pucker. The conformational parameters defining the sugar geometries are the two endocyclic torsions $\nu_{1}$ and $\nu_{2}$ about $\mathrm{C}^{\prime}$ - $\mathrm{Cl}^{\prime}$ and $\mathrm{C}^{\prime}$-C2' bonds, respectively. However, in Tables II-V, the sugar geometries have been expressed in terms of the more well known parameters ${ }^{12}$, phase angle $(\varphi)$ and the extent of pucker $\left(\tau_{m}\right)$. In what follows, an account of the investigations on dinucleoside monophosphates, with a few base sequences, will be presented.

\section{METHOD OF CALCULATION}

In evaluating the energies of dinucleoside monophosphates, LennardJones potential function for nonbonded interactions, Coulomb potential for electrostatic interactions, and three-fold potential for torsional strain were employed ${ }^{13}$. The nonbonded parameters used in the investigations are as listed, previously ${ }^{13}$, with the phosphate charges taken from Ref. 14. The sugar geometries corresponding to various pucker forms were chosen, as before ${ }^{10}$, from the energetically favorable regions of the pseudorotational space for 2'- $\beta$-D-deoxyribose ${ }^{7}$. The conformational energies were minimized with respect to the torsions $\alpha$, $\beta, \gamma, \epsilon, \zeta$, and $\chi$, for each of the sugar geometries corresponding to a given pucker. The minimum of such energies is used in the subsequent analysis and is tabulated.

We point out at this stage that the minimization procedure used is only a refinement procedure and not one to obtain all the possible energetically favorable local minima of the system under investigation. Therefore, we have chosen several starting sets of parameters to obtain the possible energy-minimized conformers within the framework of the conformational domain. The choice of the relevant conformational parameters is described in the following section. In the present investigation, the minimization procedure used does not distinguish two conformational states differing in energy by less than $0.05 \mathrm{kcal} / \mathrm{mol}$.

For evaluation of electrostatic energies, following Pattabiraman? the dielectric constant used for base-base interactions was kept at 1 . Note that under the conditions of base stacking, as in a regular doublehelical base-paired polynucleotide, the electrostatic interactions between the base atoms are not influenced by the environment (e.g., solvent or counterions) of the nucleotides. The dielectric constant for the rest of the interactions was kept at 4 . This value was earlier shown 
to be "quite reasonable" for evaluation of electrostatic interactions between static charges, in the environments obtainable in biological systems (see Ref. 13). Several investigations on the conformations of nucleic acids and their fragments (referenced in this paper) have employed this value. Use of distance-dependent dielectric constants, as by Kollman and coworkers in their investigations on polynucleotides and polypeptides ${ }^{15}$, are not likely to alter the overall qualitative consistency of the results obtained.

In the present investigation, four base sequences- $d(\mathrm{GpC}), \mathrm{d}(\mathrm{CpG})$, $\mathrm{d}(\mathrm{Ap} T)$, and $\mathrm{d}(\mathrm{TpA})$ - have been considered. These purine-pyrimidine and pyrimidine-purine sequences are significant as they contain the complementary bases in the Watson-Crick base-pairing scheme. Besides, several oligonucleotides, whose crystal structures have been recently studied and reported, contain these sequences. ${ }^{16-26}$

\section{CHOICE OF THE CONFORMATIONAL PARAMETERS}

As mentioned in the previous section, the limitations imposed by the minimization procedure employed necessitate the choice of a number of starting conformations to obtain an insight into the possible low-energy conformations of dinucleoside monophosphates. Therefore, the starting set of conformational parameters of these compounds were obtained following the stereochemical guidelines for the formation of double-helical structures of polynucleotides, outlined in our laboratory. ${ }^{27,28}$ These guidelines define the interrelationships between various backbone torsion angles from the point of view of helical structures with $n$ (number of residues per turn of the helix) between 8 and 12 and $h$ (the unit height) between 2.5 and $4.0 \AA$. It has been pointed out that only a limited number of classes of conformational combinations leads to helical structures with the above ranges of $n$ and $h$. Table I lists such combinations of the backbone torsion angles. The starting values for these parameters are also indicated in Table I. Note that the combination (gauche-, $\mathrm{E}^{3}$, trans, (gauche-, gauche $\left.{ }^{+}\right)$, trans) [that is, $\gamma$ (gauche -), $\delta\left(\mathrm{E}^{3}\right.$ or $\mathrm{C}^{\prime}-$ endo), $\epsilon$ (trans), $\zeta$ (gauche ${ }^{-}$), $\alpha$, (gauche ${ }^{+}$), and $\beta$ (trans)] has not been considered, as earlier studies on model compounds ${ }^{29}$ have shown to correspond to energetically unfavorable structures. In later tests, the helical domains have been referred to in terms of sugar pucker and phosphodiester conformations, as was done earlier. ${ }^{27}$

In base-paired double-helical structures, the classification of $\chi$ values in the anti, syn, high-anti, and low-anti ranges is influenced to a certain extent by sugar geometries, as pointed out earlier ${ }^{11}$. While lowand high-anti values of $\chi$ were shown to correspond to left-handed base-paired double-helical structures, the other two ranges corresponded to right-handed structures ${ }^{11}$. In the present study, the starting values for $\chi$ in the anti, high-anti, syn, and low-anti ranges were $230^{\circ}$, 
TABLE I

Conformational Combinations of the Backbone Torsion Angles, Meaningful from the Viewpoint of Double-Helical Base-Paired Structures of Polynucleotides. ${ }^{a}$

\begin{tabular}{|c|c|c|c|c|c|c|}
\hline No. & $\epsilon$ & $\xi$ & $\alpha$ & $\beta$ & $\gamma$ & $\delta$ \\
\hline I & $t(200)$ & $g^{-}$ & $g^{-}$ & $t\left(180^{\circ}\right)$ & $g^{+}\left(60^{\circ}\right)$ & C3'-endo \\
\hline II & $t$ & $t$ & $g^{-}$ & $t$ & $g^{+}$ & $\mathrm{C} 2^{\prime}$-endo \\
\hline III & $t$ & $g^{-}$ & $t$ & $t$ & $t\left(180^{\circ}\right)$ & C3'-endo \\
\hline IV & $t$ & $t$ & $t$ & $t$ & $t$ & $\mathrm{C} 2$-endo \\
\hline V & $t$ & $t$ & $g^{+}$ & $t$ & $g^{-}\left(300^{\circ}\right)$ & $\mathrm{C} 2$-endo \\
\hline VI & $g^{-}\left(280^{\circ}\right)$ & $t$ & $t$ & $t$ & $g^{+}$ & $\mathrm{C} 2$-endo \\
\hline VII & $g^{-}$ & $t$ & $g^{+}$ & $t$ & $t$ & $\mathrm{C} 2$-endo \\
\hline
\end{tabular}

${ }^{a} g^{-}, g^{+}$, and $t$ stand for gauche-, gauche ${ }^{+}$, and trans conformations, respectively. The numbers within the paranthesis represent the starting values (in degrees) of the torsion angle parameters. For example, the starting value for $\gamma$ in the $g^{+}, t$, and $g^{-}$ configurations are $60^{\circ}, 180^{\circ}$, and $300^{\circ}$, respectively. The phosphodiester conformations were varied by $30^{\circ}$ around their standard conformations $\left(\mathrm{g}^{+}=60^{\circ}, t=180^{\circ}\right.$, and $g^{-}=300^{\circ}$ ). Thus, in each helical domain, energies were minimized for nine combinations of $\xi$ and $\alpha$. For example, in helical domain $I$, the nine combinations considered were $\left(270^{\circ}, 270^{\circ}\right),\left(270^{\circ}, 300^{\circ}\right),\left(270^{\circ}, 330^{\circ}\right),\left(300^{\circ}, 270^{\circ}\right),\left(300^{\circ}, 300^{\circ}\right),\left(300^{\circ}, 330^{\circ}\right),\left(330^{\circ}, 270^{\circ}\right)$, $\left(330^{\circ}, 300^{\circ}\right),\left(330^{\circ}, 330^{\circ}\right)$. Of the nine values of minimized energies, the lowest was considered to represent the corresponding helical domain.

$290^{\circ}, 50^{\circ}$, and $160^{\circ}$ respectively. However, at these $\chi$ values, the handedness of the polynucleotide helix is definitely left- or right-handed, independent of the sugar pucker ${ }^{11}$.

\section{$\mathrm{d}(\mathrm{GpC})$}

In the case of $d(\mathrm{GpC})$, energy-minimization studies were carried out in the seven helical domains (listed in Table $\mathrm{I}$ ) for two broad ranges of $\chi$, namely, anti and syn. Table II lists the energetically most favorable of the conformers, corresponding to the anti and low-anti orientations of the bases, for each of the seven helical domains examined.

The global minimum corresponds to $\left(\mathrm{C} 2\right.$ '-endo $\left.\mathrm{tg}^{-}\right)$helical domain (Fig. 2). Conformers with $\mathrm{C} 2$ '-endo sugar puckers have $\chi$ values higher than those with $\mathrm{C}^{\prime}$-endo sugar puckers by about $20^{\circ}$. This is in agreement with the general relationship between the sugar pucker and glycosidic orientations in relation to base-paired double-helical structures of polynucleotides ${ }^{11}$. Conformers corresponding to helical domains I, II, III, and IV lie within $5 \mathrm{kcal} / \mathrm{mol}$ of the global minimum. On the other hand, the conformers corresponding to the other three domains listed in Table I are more than $5 \mathrm{kcal} / \mathrm{mol}$ above the global minimum. In the light of these calculations, it is found that the general trend of conformers with $\mathrm{C} 2$ '-endo sugars having higher $\chi$ values than those with $\mathrm{C} 3$ '-endo sugars is maintained. Also, a large number of 
TABLE II

Energetically Favorable Conformers of $\mathrm{d}(\mathrm{GpC})$ Corresponding to Anti and Low-anti Orientations of the Bases

\begin{tabular}{rrrrrrrrrrr}
\multicolumn{1}{c}{$\varphi$} & \multicolumn{1}{c}{$\tau_{m}$} & \multicolumn{1}{c}{$\delta$} & \multicolumn{1}{c}{$\gamma$} & \multicolumn{1}{c}{$\xi$} & \multicolumn{1}{c}{$\alpha$} & $\beta$ & \multicolumn{1}{c}{$\begin{array}{c}\text { Energy } \\
(\mathrm{kcal} / \mathrm{mol})\end{array}$} & $\begin{array}{r}\text { Helical } \\
\text { Domain }\end{array}$ \\
\hline 19.5 & 42.0 & 84.7 & 63 & 183 & 297 & 277 & 175 & 218 & -51.4 & $\mathrm{I}$ \\
160.5 & 36.4 & 136.0 & 49 & 179 & 244 & 311 & 174 & 239 & $-52.1^{\mathrm{a}}$ & $\mathrm{II}$ \\
358.0 & 39.4 & 92.8 & 159 & 189 & 296 & 168 & 176 & 204 & -48.4 & $\mathrm{II}$ \\
177.0 & 43.7 & 150.6 & 181 & 193 & 243 & 142 & 187 & 229 & -49.7 & $\mathrm{IV}$ \\
176.4 & 34.6 & 139.5 & 284 & 190 & 247 & 50 & 188 & 238 & -45.4 & $\mathrm{~V}$ \\
143.5 & 37.2 & 123.3 & 62 & 292 & 168 & 199 & 168 & 232 & -47.2 & VI \\
177.0 & 43.7 & 150.6 & 159 & 286 & 209 & 80 & 179 & 229 & -44.9 & VII \\
178.0 & 39.4 & 147.9 & 72 & 194 & 188 & 309 & 161 & 199 & -47.7 & $\mathrm{II}^{\mathrm{b}}$ \\
177.0 & 43.7 & 150.6 & 73 & 185 & 218 & 328 & 212 & 204 & -46.6 & $\mathrm{II}^{\mathrm{b}}$ \\
& & & & & & & & & & \\
158.5 & 32.0 & 133.2 & 69 & 183 & 192 & 300 & 160 & 204 & -46.4 & $\mathrm{II}^{\mathrm{b}}$ \\
& & & & & & & & & & \\
\hline
\end{tabular}

ainimum energy conformation.

${ }^{b}$ Conformers with low-anti orientations of the bases.

conformers with both $\mathrm{C} 2$ '-endo and $\mathrm{C}^{\prime}$ '-endo sugar puckers lie within $5 \mathrm{kcal} / \mathrm{mol}$ of the global minimum. In view of these facts, further calculations on $\mathrm{d}(\mathrm{GpC})$ have been restricted to the helical domains $\left(\mathrm{C}^{\prime}\right.$-endo $\left.\mathrm{tg}^{-}\right)$and $\left(\mathrm{C3}^{\prime}\right.$-endo $\left.\mathrm{g}^{-} \mathrm{g}^{-}\right)$only. Earlier studies ${ }^{30}$ by Broyde and coworkers suggested that conformers with $\mathrm{C} 3$ '-endo sugar puckers constitute the energetically most favored arrangement for $\mathrm{d}(\mathrm{GpC})$, in contrast to the corresponding feature of the present study.

Conformers with low-anti orientations of the bases and C2'-endo sugar puckers are found to be about 8 to $10 \mathrm{kcal} / \mathrm{mol}$ above the global minimum. These conformers have $\chi$ values in the range of $186^{\circ}-204^{\circ}$.
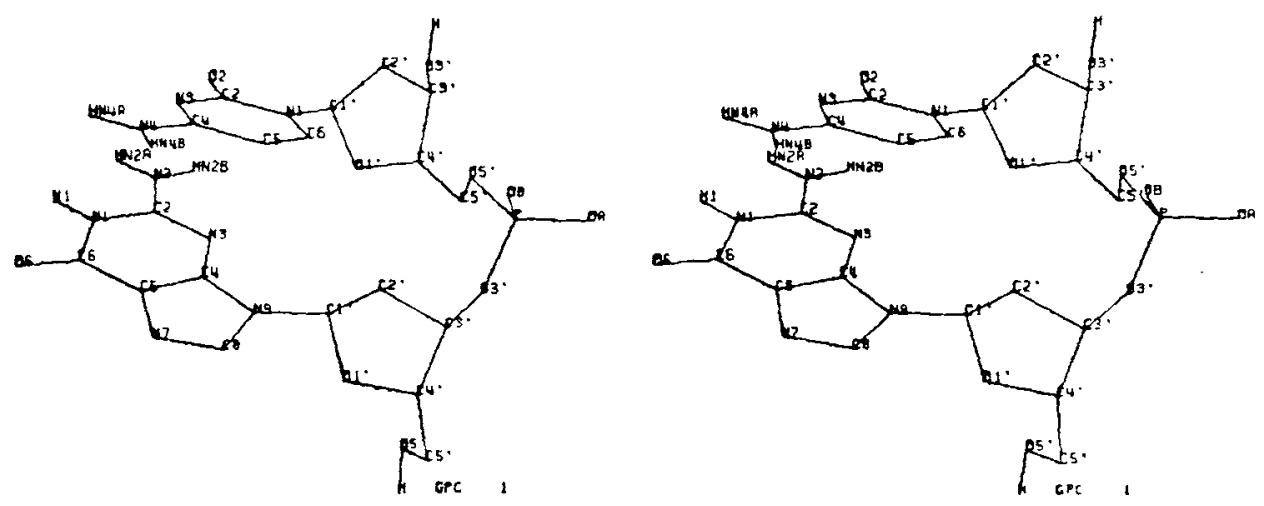

Fig. 2. Stereo pair of the global minimum conformation of $\mathrm{d}(\mathrm{GpC})$. 
Energy minimization of conformers with (C3'-endo high-anti) combinations of sugar pucker and base orientations led to arrangements that are destablized by more than $15 \mathrm{kcal} / \mathrm{mol}$ relative to the global minimum. Hence, they have not been listed.

Energy minimization with syn conformations of the bases with $\mathrm{C} 2$ 'endo sugar puckers led to conformers that are about $10-15 \mathrm{kcal} / \mathrm{mol}$ above the global minimum. Conformers with (C3'-endo syn) combination are destabilized by about $25 \mathrm{kcal} / \mathrm{mol}$ relative to the most preferred conformation. This is understood in the light of the fact that for pyrimidine nucleotides, this combination leads to energetically unfavorable arrangements. The conformers with syn orientations of the bases have also not been listed. It may be noted that employing any other helical domain in the above calculations (that is, with low-anti, high-anti, and syn conformations of the bases) would still lead to the energy differences of the above order.

\section{$\mathbf{d}(\mathbf{C p G})$}

Unlike the case of $d(\mathrm{GpC})$, energy minimization of $d(\mathrm{CpG})$ was restricted to the first four helical domains, listed in Table I, for reasons indicated in the previous section. Note that energy minimization of conformers starting with low-anti orientations of the bases invariably led to final conformers with either anti or high-anti orientations for both C2'-endo and C3'-endo sugar puckers. The global minimum has a (C3'- endo high-anti) combination of the sugar pucker and glycosidic orientation (Fig. 3). Conformers with C2'-endo sugar puckers (in the helical domains II and IV) are at least $5 \mathrm{kcal} / \mathrm{mol} \mathrm{higher} \mathrm{in} \mathrm{energy}$ than the global minimum. Table III lists a few conformers of $d(C p G)$, corresponding to both $\mathrm{C}^{\prime}$-endo and $\mathrm{C} 2$ '-endo sugars.
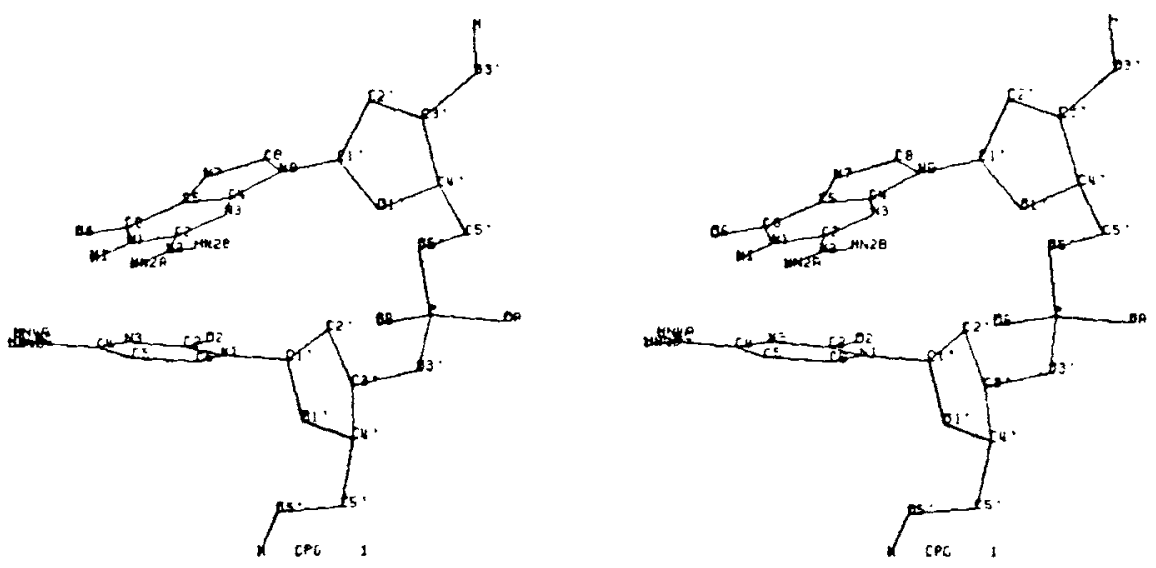

Fig. 3. Stereo pair of the global minimum conformation of $d(C p G)$. 
TABLE III

Energetically Favorable Conformers of $\mathrm{d}(\mathrm{CpG})$ with Sugar Puckers in the $\mathrm{C} 3$ '-endo and $\mathrm{C}^{\prime}$-endo Regions

\begin{tabular}{|c|c|c|c|c|c|c|c|c|c|}
\hline$\varphi$ & $\tau_{m}$ & $\delta$ & $\gamma$ & $\epsilon$ & $\xi$ & $\alpha$ & $\beta$ & $\chi$ & $\begin{array}{c}\text { Energy } \\
(\mathrm{kcal} / \mathrm{mol})\end{array}$ \\
\hline \multicolumn{10}{|c|}{ C3'-endo } \\
\hline 17.5 & 31.5 & 94.3 & 61 & 186 & 281 & 292 & 167 & 262 & $-46.1^{\mathrm{a}}$ \\
\hline 17.5 & 31.5 & 94.3 & 184 & 183 & 286 & 146 & 189 & 270 & -44.1 \\
\hline 34.7 & 42.1 & 83.2 & 71 & 188 & 284 & 290 & 161 & 263 & -43.2 \\
\hline 39.4 & 39.0 & 86.6 & 73 & 188 & 284 & 286 & 163 & 262 & -42.6 \\
\hline 38.1 & \multicolumn{7}{|c|}{ C2'-endo } & & -42.6 \\
\hline 177.0 & 30.0 & 136.6 & 169 & 194 & 224 & 148 & 223 & 251 & -40.1 \\
\hline 158.5 & 32.0 & 133.2 & 64 & 178 & 255 & 301 & 164 & 255 & -38.2 \\
\hline 139.5 & 33.3 & 121.8 & 60 & 191 & 275 & 288 & 170 & 247 & -37.9 \\
\hline 146.3 & 29.9 & 123.5 & 56 & 187 & 260 & 306 & 161 & 253 & -37.8 \\
\hline 178.0 & 39.4 & 147.9 & 172 & 192 & 239 & 148 & 205 & 247 & -37.5 \\
\hline 123.7 & 45.1 & 124.6 & 45 & 175 & 260 & 315 & 171 & 55 & $-39.8^{\mathrm{b}}$ \\
\hline 127.3 & 40.6 & 115.8 & 44 & 171 & 269 & 312 & 177 & 60 & $-39.3^{b}$ \\
\hline
\end{tabular}

s Minimum energy conformation.

${ }^{b}$ Conformers with syn orientation of the bases.

The preference for the high-anti orientations of the bases in the $\mathrm{C3}^{\prime}$-endo conformers of $\mathrm{d}(\mathrm{CpG})$ can be understood in the light of the conformational analysis of $5^{\prime}$-nucleotides ${ }^{10}$. For C 3 '-endo sugars, the anti conformation of the bases are energetically favorable for $3^{\prime}$-dCMP but not for 5'-dGMP. Also, the (C3'-endo high-anti) combinations are energetically favorable for $3^{\prime}$-dCMP and 5 -dGMP, although the preferred ranges of $\chi$ for both the compounds are not identical. Hence, minimization of energy for $d(\mathrm{CpG})$ would tend to shift the values of $\chi$ to the high-anti region. However, the values were restricted to around $260^{\circ}-270^{\circ}$. This is because the ranges of bordering high-anti and syn, such as $-40^{\circ}$ to $0^{\circ}$, are not energetically favorable for $3^{\prime}$-dCMP, although they are favored for $5^{\prime}$-dGMP.

In contrast, for $d(\mathrm{GpC})$, the anti orientations of the bases are preferred because both 3 '-dGMP and 5 '-dCMP have energetically favorable conformers in the (C3'-endo anti) and ( $\mathrm{C}^{\prime}$ '-endo anti) regions. The anti orientations of the bases in conformers with $\mathrm{C} 2$ '-endo sugars, in $\mathrm{d}(\mathrm{CpG})$ are also understood on the basis of the occurrence of secondary minima in the (C2'-endo anti) region of both $3^{\prime}$-dCMP and $5^{\prime}$-dGMP.

Minimization of energies of $\mathrm{d}(\mathrm{CpG})$ with $(\mathrm{C} 2$ '-endo syn $)$ combinations led to conformers that are about $7-10 \mathrm{kcal} / \mathrm{mol}$ above the global minimum. Two of these conformers have also been listed in Table III. Conformers with (C3'-endo syn) combinations are energetically 
destabilized by about $25 \mathrm{kcal} / \mathrm{mol}$, as in $\mathrm{d}(\mathrm{GpC})$ and hence they have not been listed.

Thus, the above-discussed calculations indicate that the global minimum of $d(\mathrm{GpC})$ (corresponding to helical domain II) is lower in energy than that of $\mathrm{d}(\mathrm{CpG})$ (corresponding to helical domain I) by about 6 $\mathrm{kcal} / \mathrm{mol}$ (see Tables II and III). The former has anti orientations of the bases, as against the high-anti orientations in the latter. In the case of $\mathrm{d}(\mathrm{GpC})$, a large number of conformers with both $\mathrm{C3}^{\prime}$-endo and C2'-endo sugars lie within $5 \mathrm{kcal} / \mathrm{mol}$ of its global minimum. However, in $\mathrm{d}(\mathrm{CpG})$, the $\mathrm{C} 2{ }^{\prime}$-endo conformers are at least $5 \mathrm{kcal} / \mathrm{mol}$ above the corresponding global minimum.

\section{$d(\mathbf{A p T})$ and $d(\mathbf{T p A})$}

As indicated in the earlier section $d(\mathrm{GpC})$, energy minimizations of $\mathrm{d}(\mathrm{ApT})$ and $\mathrm{d}(\mathrm{TpA})$ were restricted to the helical domains I-IV. Also, in light of the results discussed in the two previous sections, the base conformations were restricted to the anti range of $\chi$ only. Tables IV and $V$ list a few of the energetically favorable conformers of $d(A p T)$ and $\mathrm{d}(\mathrm{TpA})$, respectively.

In $\mathrm{d}(\mathrm{Ap} \mathrm{T})$, as in $\mathrm{d}(\mathrm{GpC})$, the global minimum corresponds to helical domain II and has a $\chi$ value of $243^{\circ}$ (Fig. 4). The most preferred of the conformers with sugar puckers in the C $3^{\prime}$-endo region is about $1 \mathrm{kcal} /$ mol higher in energy than the global minimum with $\mathrm{C} 2$ '-endo sugar. This result is in sharp contrast to that reported by earlier studies, ${ }^{31}$ which had incorporated the rigid-nucleotide concept. Conformers with C3'-endo sugars were shown to be energetically favored over those

TABLE IV

Energetically Favorable Conformers of d(ApT) with Sugars in the C2'endo and $\mathrm{C} 3$ '-endo Regions

\begin{tabular}{|c|c|c|c|c|c|c|c|c|c|}
\hline$\varphi$ & $\tau_{m}$ & $\delta$ & $\gamma$ & $\epsilon$ & $\xi$ & $\alpha$ & $\beta$ & $\chi$ & $\begin{array}{c}\text { Energy } \\
(\mathrm{kcal} / \mathrm{mol})\end{array}$ \\
\hline \multicolumn{10}{|c|}{$\mathrm{C} 2$-endo } \\
\hline 155.5 & 42.9 & 135.8 & 60 & 178 & 247 & 301 & 171 & 243 & $-42.4^{a}$ \\
\hline 156.9 & 47.0 & 138.5 & 57 & 181 & 244 & 301 & 173 & 242 & -41.3 \\
\hline 160.5 & 36.4 & 136.0 & 50 & 174 & 251 & 307 & 179 & 246 & -42.0 \\
\hline 158.5 & 32.0 & 133.2 & 51 & 172 & 254 & 306 & 179 & 247 & -41.0 \\
\hline 178.0 & 39.4 & 147.9 & 171 & 178 & 235 & 163 & 201 & 234 & -39.1 \\
\hline 177.0 & 43.7 & 150.6 & 168 & 179 & 240 & 168 & 191 & 233 & -38.9 \\
\hline \multicolumn{10}{|c|}{ C3'endo } \\
\hline 19.5 & 42.0 & 84.7 & 60 & 188 & 295 & 283 & 171 & 220 & -41.3 \\
\hline 14.1 & 35.9 & 91.2 & 64 & 179 & 295 & 275 & 176 & 228 & -40.5 \\
\hline 16.7 & 45.7 & 81.3 & 60 & 186 & 297 & 284 & 172 & 216 & -40.4 \\
\hline 17.5 & 31.5 & 94.3 & 53 & 176 & 286 & 290 & 174 & 235 & -39.1 \\
\hline 356.4 & 34.6 & 96.8 & 161 & 194 & 291 & 160 & 189 & 200 & -38.6 \\
\hline 357.0 & 30.0 & 100.6 & 161 & 193 & 290 & 160 & 187 & 200 & -38.2 \\
\hline
\end{tabular}

Minimum energy conformation. 
TABLE V

Energetically Favorable Conformers of $\mathrm{d}(\mathrm{TpA})$ with $\mathrm{C3}^{\prime}$-endo and $\mathrm{C2}$ '-endo Sugars

\begin{tabular}{|c|c|c|c|c|c|c|c|c|c|}
\hline$\varphi$ & $\tau_{m}$ & $\delta$ & $\gamma$ & $\epsilon$ & $\xi$ & $\alpha$ & $\beta$ & $x$ & $\begin{array}{c}\text { Energy } \\
(\mathrm{kcal} / \mathrm{mol})\end{array}$ \\
\hline \multicolumn{10}{|c|}{ C3'-endo } \\
\hline 51.3 & 32.2 & 93.6 & 51 & 190 & 271 & 280 & 170 & 231 & $-44.1^{\mathrm{a}}$ \\
\hline 357.0 & 30.0 & 100.6 & 65 & 187 & 281 & 279 & 172 & 245 & -41.0 \\
\hline 14.1 & 35.9 & 91.2 & 56 & 183 & 288 & 289 & 171 & 233 & -40.4 \\
\hline 17.5 & 31.5 & 94.3 & 56 & 178 & 289 & 287 & 176 & 242 & -39.6 \\
\hline 356.4 & 34.6 & 96.8 & 65 & 186 & 285 & 277 & 173 & 240 & -39.4 \\
\hline \multicolumn{10}{|c|}{ C2'-endo } \\
\hline 155.5 & 42.9 & 135.8 & 58 & 184 & 242 & 297 & 169 & 248 & -38.2 \\
\hline 156.9 & 47.0 & 138.5 & 59 & 179 & 245 & 299 & 171 & 248 & -37.8 \\
\hline 160.5 & 36.4 & 136.0 & 61 & 182 & 245 & 292 & 173 & 252 & -38.2 \\
\hline 178.0 & 39.4 & 147.9 & 166 & 183 & 226 & 159 & 212 & 241 & -36.8 \\
\hline 176.4 & 34.6 & 139.5 & 163 & 180 & 237 & 166 & 204 & 240 & -36.7 \\
\hline
\end{tabular}

a Minimum energy conformation.

with $\mathrm{C} 2$ '-endo sugars by $3 \mathrm{kcal} / \mathrm{mol}^{31}$. Besides, these studies were not carried out in the framework of regular helical structures. In d(ApT), too, the trend of lower $\chi$ values for conformers with $\mathrm{C}^{\prime}$-endo sugars than in those with $\mathrm{C}^{\prime}$-endo sugars is observed.

As in $\mathrm{d}(\mathrm{CpG})$, the global minimum of $\mathrm{d}(\mathrm{TpA})$ has sugar pucker in the C3'-endo region (Fig. 5). However, the glycosidic orientations in the two pyrimidine-purine sequences are different. For $d(T p A)$, the global minimum has $\chi$ equal to $231^{\circ}$. Note that for both $3^{\prime}$-dTMP and 5 -dAMP, the C3'-endo anti conformational combination constitutes energetically favored arrangements. Hence, minimization of energy of $\mathrm{d}(\mathrm{TpA})$ in the anti range of $\chi$ leads to a resultant value of $\chi$ also in the anti range.
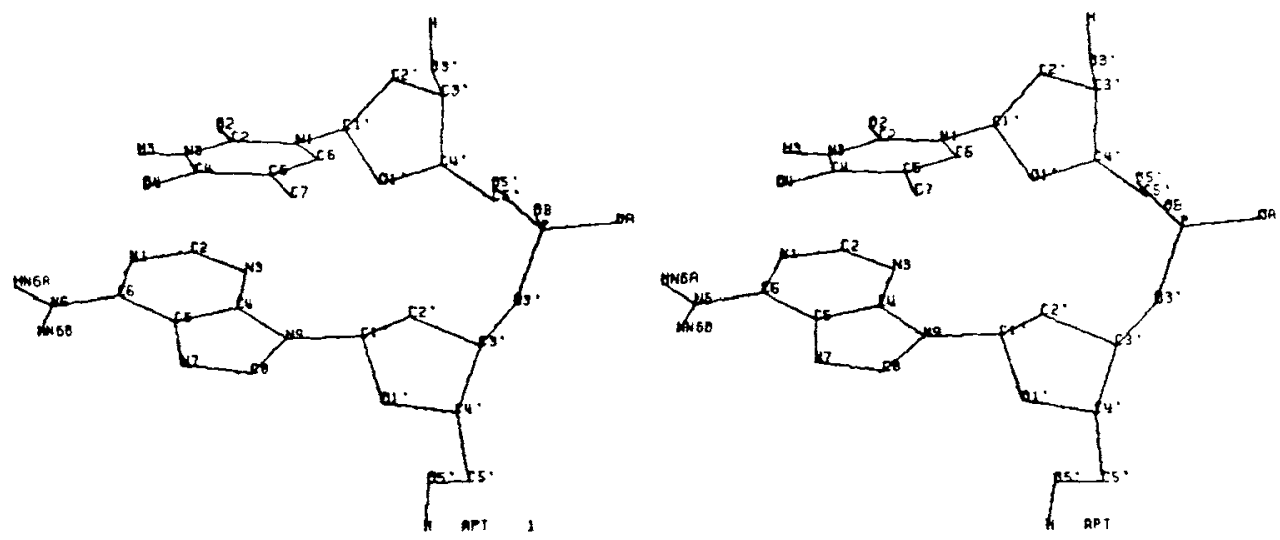

Fig. 4. Stereo pair of the global minimum conformation of $d(A p T)$. 

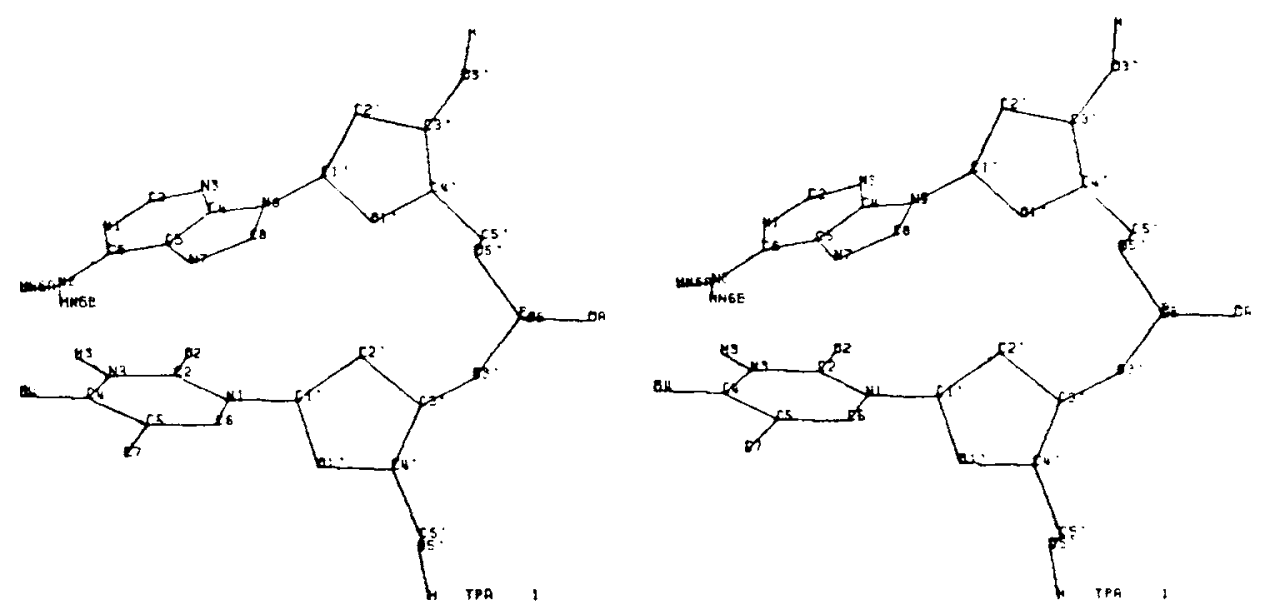

Fig. 5. Stereo pair of the global minimum conformation of d(ApT).

Summarizing, the global minima of the purine-pyrimidine sequences correspond to the ( $\mathrm{C2}^{\prime}$-endo $\left.\mathrm{tg} \mathrm{g}^{-}\right)$helical domain, and those of the pyrimidine-purine sequences correspond to the ( $\mathrm{C}^{\prime}$-endo $\left.\mathrm{g}^{-} \mathrm{g}^{-}\right)$ helical domain. For the former, a number of conformers with both $\mathrm{C} 2$ 'endo and $\mathrm{C3}^{\prime}$-endo sugar puckers lie within $5 \mathrm{kcal} / \mathrm{mol}$ of the global minimum. In the latter, the $\mathrm{C} 2$ '-endo conformers are at least $5 \mathrm{kcal} /$ mol higher in energy than the global minimum. The calculations emphasize the significance of sugar-ring flexibility and demonstrate the effect of base-base stacking interactions on the mononucleotide conformations. For example, while the global minima of $3^{\prime}$-dGMP and 5 dCMP, have respectively syn and anti orientations of the bases, in $\mathrm{d}(\mathrm{GpC})$, the global minimum has anti orientations of the bases. Also, in $\mathrm{d}(\mathrm{CpG})$, the global minimum has high-anti orientations of the bases $\left(\chi \sim 260^{\circ}\right)$. In contrast, the energetically most preferred conformers of $3^{\prime}$-dCMP and $5^{\prime}$-dGMP have anti $\left(\sim 210^{\circ}\right)$ and high-anti-syn $\left(\sim 330^{\circ}\right)$ ranges of $\chi$, respectively, together with sugar puckers in the $\mathrm{C} 3{ }^{\prime}$-endo region. The salient features of these calculations are unlikely to be affected by the inclusion of anomeric effect, as they result from the properties of base-base stacking interactions.

\section{DISCUSSION AND CONCLUSION}

The present investigations have a few interesting implications for understanding polynucleotide helical structures found thus far by $\mathrm{x}$-ray diffraction methods. Note that the global minimum conformation of $\mathrm{d}(\mathrm{GpC})$ corresponds to a right-handed helical structure with $n$ and $h$ values close to those of the conventional uniform helical forms such as $\mathrm{A}, \mathrm{B}$, and $\mathrm{C}$ (the helical parameters were evaluated following the method of Sugeta and Miyazawa ${ }^{32}$ ). Also, the low-anti conformers correspond to left-handed helical structures with the $n$ and $h$ values 
similar to those of the right-handed structures. On the other hand, the global minimum conformer of $\mathrm{d}(\mathrm{CpG})$ corresponds to a left-handed helical structure with low $h$ value $(<1 \AA)$ and high $n$ values $(\sim 14)$. This is consistent with earlier model-building studies, ${ }^{11}$ which had indicated that double-helical polynucleotide models with high-anti orientations of the bases had large radii, which result from such small $h$ values as $1 \AA$.

Conformers with $\mathrm{C} 2$ '-endo sugars correspond to right-handed helical structures with $n$ and $h$ values close to those of the conventional uniform helical forms. Thus, both left- and right-handed base-stacking arrangements are possible for $\mathrm{d}(\mathrm{GpC})$ and $\mathrm{d}(\mathrm{CpG})$, with the proper choice of the base orientations, vindicating the earlier classification of double-helical structures in the $\chi$-space ${ }^{11}$. Therefore, polynucleotides containing the sequence (...CGCGCG....) would tend to exhibit both right- and left-handed helical forms. However, this feature is in contrast to that obtained by earlier investigations on base-base stacking interactions, which concluded that $\mathrm{d}(\mathrm{GpC})$ and $\mathrm{d}(\mathrm{CpG})$ could stack only in right- and left-handed helical senses, respectively. $7,33,34$

The global minima of both $d(\mathrm{ApT})$ and $\mathrm{d}(\mathrm{TpA})$ correspond to righthanded uniform helical structures with $n$ and $h$ values similar to those of the commonly occurring forms, while the secondary minima of these two compounds with low-anti orientations of the bases correspond to left-handed uniform helical structures. This implies that sequences of the type (...ATATAT....) would readily take up uniform right- and left-handed helical structures such as A, B, C, and D. Such structures could be built with both the $\mathrm{C} 2$ '-endo and the $\mathrm{C} 3$ '-endo sugar puckers. They would be respectively associated with $\left(\mathrm{tg}^{-}\right)$and $\left(g^{-} g^{-}\right)$phosphodiester conformations, as pointed out earlier. ${ }^{35,36}$ However, the right-handed form will be energetically favored over the lefthanded form for polynucleotides containing large stretches of $\mathrm{A}$ and $\mathrm{T}$ bases.

Thus, our studies on the dinucleoside monophosphates in the framework of uniform helical structures (with mononucleotide repeat) indicate that while the global minimum conformers of $\mathrm{d}(\mathrm{GpC}), \mathrm{d}(\mathrm{ApT})$, and $\mathrm{d}(\mathrm{TpA})$ tend to promote helical structures of the forms such as $\mathrm{A}$, $B, C$, and $D$, that of $d(C p G)$ tends to break or perturb such helical structures. Further, the significance of conformers that constitute secondary minima is also demonstrated in the light of double-helical polynucleotide conformations. For example, the (C2'-endo anti) conformational combination that is associated with the B-DNA structure is shown to be present in several synthetic polynucleotides, both in solution $^{37-39}$ and in crystals. ${ }^{20,25}$ In such structures, the energy differences between the above conformational combination and the global minimum conformation of the dinucleoside monophosphates (in the framework of uniform helical structures) are compensated by other stabilizing interactions, such as hydrogen bonding (base-pairing), 
stacking between adjacent base pairs, and crystal packing forces. The influence of such forces could be understood through conformational analysis of base-paired dinucleoside monophosphate moieties and their derivatives, which would throw further light on the structural aspects of base-paired double-helical polynucleotides of varying sequences. Such investigations are in progress in our laboratory and will be discussed elsewhere.

We thank the Department of Science and Technology, Government of India for the SERC Research Grant that supported this work. We also thank Dr. N.V. Joshi, Center for Theoretical Studies, Bangalore, India, and Professor Peter Kollman and Dr. Terry Lybrand of the Department of Pharmaceutical Chemistry, University of California, San Francisco, for extremely useful discussions and invaluable suggestions. (S.N.R.) thanks the Council for Scientific and Industrial Research, India, for a fellowship.

\section{References}

1. Yathindra, N. \& Sundaralingam, M. (1973) Biopolymers 12, 287-314.

2. Yathindra, N. \& Sundaralingam, M. (1973) Biopolymers 12, 2075-2082.

3. Yathindra, N. \& Sundaralingam, M. (1973) Biopolymers 12, 2261-2277.

4. Pullman, B. \& Saran, A. (1976) Prog. Nucleic Acid Res. Mol. Biol. 18, 215-322.

5. Ts'O, O. P. P. (1974) in Basic Principles in Nucleic Acid Chemistry, Vols. 1 and 2, Academic, New York.

6. Bloomfield, V. A., Crothers, D. M. \& Tinoco, Jr., I. (1974) in Physical Chemistry of Nucleic Acids, Harper \& Row, New York.

7. Pattabiraman, N. (1979) Ph.D. Thesis, Indian Institute of Science, Bangalore, India.

8. Sundaralingam, M. (1973) in Conformation of Biological Molecules and Polymers (5th Jerusalem Symp. Quant. Chem. Biochem.) Bergmann, E. D. \& Pullman, B., Eds., Academic, New York, pp. 417-455.

9. Pattabiraman, N., Rao, S. N. \& Sasisekharan, V. (1980) Nature 284, 187-189.

10. Rao, S. N. \& Sasisekharan, V. (1981) Ind. J. Biochem. Biophys. 18, 303-310.

11. Rao, S. N. \& Sasisekharan, V. (1983) Int. J. Biol. Macromol.5, 83-88.

12. Altona, C. \& Sundaralingam, M. (1972) J. Am. Chem. Soc. 94, 8205-9212.

13. Ramachandran, G. N. \& Sasisekharan, V. (1968) Adv. Protein Chem. 23, 283-437.

14. Sasisekharan, V. \& Lakshminarayanan, A. V. (1969) Biopolymers 8, 505-514.

15. Weiner, S. J., Kollman, P. A., Case, D., Singh, U. C., Ghio, C., Alagona, G., Profeta, S., Jr. \& Weiner, P. K. (1983) J. Am. Chem. Soc. 106, 765-784.

16. Crawford, J. L., Kolpak, F. J., Wang, A. H.J., Quigley, G. J., van Boom, J. H., van der Marel, G. \& Rich, A. (1980) Proc. Natl. Acad. Sci. USA 77, 4016-4020.

17. Drew, H. R., Takano, T., Tanaka, S., Itakura, K. \& Dickerson, R. F. (1980) Nature 286, 567-573.

18. Wang, A. H.-J., Quigley, G. J., Kolpak, F. J., Crawford, J. L., van Boom, J. H., van der Marel, G. \& Rich, A. (1979) Nature 282, 680-686.

19. Kopka, M. L., Fratini, A. V., Drew, H. R. \& Dickerson, R. E. (1983) J. Mol. Biol. $163,129-146$.

20. Dickerson, R. E. (1983) J. Mol. Biol. 166, 419-441.

21. Wang, A. H.-J., Fujii, S., van Boom, J. H. \& Rich, A. (1982) Proc. Natl. Acad. Sci. USA 79, 3968-3972.

22. Viswamitra, M. A., Kennard, O., Shakked, Z., Jones, P. G., Sheldrick, G. M., Salisbury, S. A. \& Falvello, L. (1978) Nature 273, 687-688.

23. Wing, R., Drew, H. R., Takano, T., Broka, C., Tanaka, S., Itakura, K. \& Dickerson, R. E. (1980) Nature 287, 755-758.

24. Shakked, Z., Rabinovich, D., Cruse, W. B. T., Egert, E., Kennard, O., Sala G., 
Salisbury, S. A. \& Viswamitra, M. A. (1981) Proc. Roy. Soc. London, Ser. B 213, 479487.

25. Shakked, Z., Rabinovich, D., Kennard, O, Cruse, W. B. T., Salisbury, S. A. \& Viswamitra, M. A. (1983) J. Mol. Biol. 166, 183-201.

26. Viswamitra, M. A., Shakked, Z., Jones, P. G., Sheldrick, G. M., Salisbury, S. A.

\& Kennard, O. (1982) Biopolymers 21, 513-533.

27. Sasisekharan, V. \& Pattabiraman, N. (1978) Nature 275, 159-162.

28. Rao, S. N. \& Sasisekharan, V. (1982) in conformations in Biology, Srinivasan, R. \& Sarma, R.H., Eds., Adenine, New York, pp.323-334.

29. Sasisekharan, V. \& Laskshminarayanan, A. V. (1969) Biopolymers 8, 505-514.

30. Broyde, S., Wartell, R. M. \& Stellman, S. T. (1978) Biopolymers 17, 1485-1506.

31. Ponnuswamy, P. K. \& Thiagarajan, P. (1981) Biophys. J. 35, 731-752.

32. Sugeta, H. \& Miyazawa, T. (1967) Biopolymers 5, 673-679.

33. Gupta, G. \& Sasisekharan, V. (1978) Nucl. Acids Res. 5, 1639-1654.

34. Gupta, G. \& Sasisekharan, V. (1978) Nucl. Acids Res. 5, 1655-1673.

35. Gupta, G., Bansal, M. \& Sasisekharan, V. (1980) Int. J. Biol. Macromol. 2, 368380 .

36. Sasisekharan, V. \& Gupta, G. (1980) Curr. Sci. 49, 43-48.

37. Leslie, A. G. W., Arnott, S., Chandrasekaran, R. \& Ratliff, R. L. (1980) J. Mol. Biol. 143, 49-72.

38. Cohen, J. S., Wooten, J. S. \& Chatterjee, C. L. (1981) Biochemistry 20, 3049-3055.

39. Assa-Munt, N. \& Kearns, D. A. (1984) Biochemistry 23, 791-796.

40. IUPAC-IUB Joint Commission on Biochemical Nomenclature(1983) Eur. J. Biochem. $131,9-15$.

Received August 22, 1984

Accepted February 26, 1985 